\title{
CAMBIOS EN LA DINÁMICA MANDIBULAR Y EN LA CADENA SINÉRGICA MUSCULAR MANDIBULAR POSTERIOR A LA PUNCIÓN SECA Y TENS EN PACIENTE CON TTM
}

\author{
CHANGES IN MANDIBULAR DYNAMICS AND THE MANDIBULAR MUSCULAR \\ SYNERGISTIC CHAIN AFTER DRY NEEDLING AND TENS IN PATIENTS WITH TMD
}

\section{Espinoza Sebastian ${ }^{1}$, Marfull Norman ${ }^{2}$}

\section{RESUMEN}

Antecedentes: El tratamiento del trastorno temporomandibular (TTM) con la técnica de punción seca no se ha investigado a fondo. Este informe evaluó su eficacia a través del equipo BIOPAK ${ }^{\mathrm{TM}}$ (Electromiografía de Superficie (BioEMGTM) y Trazador mandibular (JT3DTM).

Presentación clínica: Paciente de 57 años con TTM grave que fue tratada previamente con un dispositivo interoclusal (DIO) de relajación, sin éxito. Se trató con la técnica de aguja seca (PS) en la parte anterior de los músculos pterigoideos lateral y medial, temporal anterior y masétero. Durante la punción, se aplicó una estimulación nerviosa eléctrica transcutánea (TENS), produciendo estimulación eléctrica neuromuscular (NMES).

Relevancia Clínica: Es importante desarrollar nuevas técnicas para casos complicados de TTM; por lo tanto, la técnica de PS más NMES podría ser una buena alternativa para restaurar la sinergia muscular normal de los músculos de la masticación.

Conclusión: Se logra una importante estabilidad biomecánica mandibular y un alivio subjetivo de los síntomas del TTM inmediatamente post PS y seis días posteriores a esta. El uso de BIOPAK ${ }^{\text {TM }}$ (BioEMGTMm y JT-3DTM), es una buena alternativa de evaluación en TTM, proporcionando mediciones fiables.
1. Universidad de Valparaíso, Escuela de Odontología, Valparaíso, Chile.

2. Universidad Andrés Bello, Escuela de Odontología, Viña del Mar, Chile.

\section{Correspondencia:}

Sebastián Espinoza. Escuela de Odontología. Subida Leopoldo Carvallo \#211, Valparaíso, Chile. CP: 2360004

Correo electrónico:

sebastian.espinoza@uv.cl

\section{PALABRAS CLAVES:}

Trastornos Temporomandibulares; Punción seca; Síndromes de Dolor Miofascial; BIOPAK $^{\mathrm{TM}}$; Caso Clínico.

\section{KEYWORDS:}

Temporomandibular Joint Disorders; Dry needling; Myofascial Pain Syndromes; BIOPAK; Case Report. 


\section{ABSTRACT}

Background: Temporomandibular disorder (TMD) treatment with the dry needling technique has not been thoroughly investigated. This report evaluated its effectiveness through Surface Electromyography (sEMG) and BioPak.

Clinical presentation: A 57-year-old female patient with severe TMD who was previously treated with a relaxing plane device without success was treated once using the dry needle technique in the anterior portion of the lateral pterygoid, medial pterygoid, and anterior portion of the temporalis and masseter muscles. During the needling, a transcutaneous electrical nerve stimulation (TENS) was applied, producing neuromuscular electrical stimulation (NMES).

Clinical Relevance: It is important to develop new techniques for complicated cases of TMD; therefore, the dry needling technique plus NMES could be a good alternative for restoring normal muscular synergy of the mastication muscles.

Conclusion: A relief of TMD symptoms and biomechanical stability was found. The use of combined sEMG and BioPak provides reliable measurements.

\section{INTRODUCCIÓN}

Los trastornos temporomandibulares (TTM) son un término colectivo para muchas alteraciones de la articulación temporomandibular (ATM) y de los músculos de la cadena masticatoria. Estos trastornos comparten síntomas y signos similares, como dolor, sonidos articulares y movimiento restringido de la mandíbula. El dolor es experimentado de manera característica en la región pre-auricular y/o en los músculos de masticación, y a menudo es concomitante con dolores de cabeza frecuentes, rechinamiento de los dientes y problemas de sueño. ${ }^{1}$

La prueba más utilizada para diagnosticar un TTM es el instrumento DC-TMD ${ }^{2}$, que es un examen clínico para las alteraciones principales de la ATM. El principal problema con la evaluación de este sistema es que es operadordependiente, incluso con clínicos entrenados. Un estudio reciente mostró buenos valores de fiabilidad para DC-TMD, pero con una falta de metodología reconocida sobre las limitaciones del estudio, lo que indica la necesidad de más investigación de confiabilidad. ${ }^{3}$ Una buena alternativa en el campo de las mediciones biométricas para establecer la función basal de activación muscular, es el equipo BIOPAK ${ }^{\mathrm{TM}}$, que ayuda a los clínicos a entender el patrón de activación de los músculos, tanto su sinergia como su simetría, junto con los rangos de movimiento de velocidad y ósteo muscular (ROM), proporcionando así evidencia de los componentes biomecánicos antes de que se produzca daño en la estructura de la ATM. Un buen examen, junto con la eliminación de otros componentes etiológicos, podría ayudar a anticipar el daño degenerativo articular. La presencia de un TTM cuando se analiza con el equipo BIOPAK ${ }^{\mathrm{TM}}$, muestra una reducción en la apertura mandibular y los rangos retrusivos, junto con los movimientos de desviación unilaterales durante el habla. ${ }^{4}$ Incluso el mioespasmo del pterigoideo lateral podría generar síntomas tales como alteraciones de la función mandibular, dificultad en el cierre después de una apertura máxima y movimientos involuntarios de la mandíbula, que pueden ser identificados por electromiografía de superficie (sEMG) ${ }^{5}$

La mayoría de las terapias utilizadas para la TTM se basan en analgésicos, con una eficacia similar con inyecciones intraarticulares y férulas oclusales. ${ }^{1}$ Una de las teorías más recientes implicaba que el rechinamiento de los dientes está relacionado con el bruxismo del sueño, pero al parecer las férulas oclusales no son eficaces para el tratamiento de este. ${ }^{6}$ Finalmente, se ha estudiado la eficacia del tratamiento de ortodoncia para TTM, pero los resultados no son concluyentes ${ }^{7}$; incluso la revisión sistemática que se realizó ha sido retirada. ${ }^{8}$

Otro método de tratamiento con nueva evidencia en varias alteraciones musculoesqueléticas es la técnica de punción seca (PS). Se ha demostrado con evidencia moderada para el manejo de los puntos gatillo miofasciales en pacientes con dolor lumbar. ${ }^{9}$ Para pacientes con dolor orofacial asociado con disfunción temporomandibular, la PS ha demostrado ser mejor que otras intervenciones para la intensidad del dolor, así como la terapia isquémica en el umbral de dolor, 
pero hay una muy baja calidad de la evidencia y un pequeño tamaño de efecto. ${ }^{10} \mathrm{La}$ PS profunda de los puntos gatillo miofasciales activos en el masetero y temporal en pacientes con TTM miofascial y bruxismo del sueño se asoció con mejoras inmediatas y de una semana en el dolor, la sensibilidad, la apertura mandibular y la discapacidad relacionada con el trastorno. ${ }^{11}$ Los parámetros clínicos son los más estudiados, pero los componentes biomecánicos aún no son claros.

\section{INFORME DEL CASO}

Se presenta una paciente de 57 años, sexo femenino, con TTM grave (Trastorno de los músculos masticadores e inflamatorio e la ATM); en la anamnesis la paciente reportó dolor bilateral en la ATM, con mayor intensidad en el lado izquierdo a la palpación, hiperactividad del temporal anterior izquierdo y los músculos maséteros superficiales, especialmente durante la mañana, y dolor cervical frecuente. La paciente es fisioterapeuta, por lo que le permite reconocer $\mathrm{y}$ describir sus síntomas con precisión. Refiriéndose al uso de férula oclusal nocturna, indica resultados relativos. La paciente también reportó crépito en ATM izquierda, movimiento limitado de la mandíbula por dolor en todos los planos, así como la presencia de una postura avanzada de la cabeza. Con esta información, el experto decidió aplicar la técnica PS con NMES y los otros clínicos utilizando el equipo BIOPAK ${ }^{\mathrm{TM}}$, llevaron a cabo la evaluación biomecánica de base. Para estos efectos la paciente firmó un consentimiento informado respetando las normas actualizadas de la declaración de Helsinski.

Cronología: El paciente fue evaluado por primera vez el 7 de octubre de 2018 por dos clínicos; el primero evaluó la biomecánica de la ATM a través de BIOPAK ${ }^{\mathrm{TM}}$, mientras que el segundo analizó los puntos gatillo miofasciales latentes. Después de esto, se aplicó PS con NMES y se midieron inmediatamente los parámetros sEMG y JT-3D. Después de seis días (6-PPS), el 13 octubre de 2018, nuevamente se evaluaron los parámetros con BIOPAK ${ }^{\mathrm{TM}}$.

Adquisición de información: Se utilizó BIOPAK $^{\mathrm{TM}}$, un conjunto de equipos diseñados por BioResearch Associates, Inc. (9275 N. 49th
Street, Suite 150 Milwaukee, WI 53223 USA), que incluye un electromiógrafo de superficie $\left(\right.$ BioEMG $\left.^{\mathrm{TM}}\right)$, un acelerómetro magnético $\left(\mathrm{JVA}^{\mathrm{TM}}\right)$ y un trazador mandibular $\left(\mathrm{JT}-3 \mathrm{D}^{\mathrm{TM}}\right)$. Esto permite a los investigadores conocer los potenciales de acción del reclutamiento de unidades motoras, describir los movimientos en 3D, la velocidad con que ejecuta las tareas y tiempo de las mismas durante el reposo mandibular, masticación, máxima intercuspidación, apriete dentario máximo, deglución, apertura, cierre y rango osteo muscular (ROM). Todos los valores de adquisición fueron registrados por Software BIOPAK $^{\mathrm{TM}}$ (Microsoft Corporation, Redmond, WA). BioEMG ${ }^{\mathrm{TM}}$ tiene ocho canales que reciben pares de señales de los músculos Temporales anteriores (TAs), Maséteros superficiales (MMs), Esternocleidoccipitomastoideos (ECOMs) y Digástricos anteriores (DA).

Protocolo de instalación: El paciente estaba cómodamente sentado con una posición natural de la cabeza, brazos relajados descansados en los muslos y sus pies en el suelo (Figura 1). El BioEMG $^{\mathrm{TM}}$ cuelga en el cuello durante el examen. En primer lugar, la piel se limpió con alcohol en cada lugar donde se colocaron los electrodos, eliminando el maquillaje, la loción, etc. En segundo lugar, los músculos a analizar se ubicaron pidiendo al paciente un apriete dentario máximo, identificando el vientre muscular, y luego los electrodos se colocaron en ambos lados para cada par de músculos, paralelos a las fibras; el electrodo de referencia se colocó en el dorso de la mano. Finalmente, todos los electrodos se conectaron al amplificador BioEMG ${ }^{\mathrm{TM}}$ con pinzas metálicas tipo caimán.

Registro Pre-PS: Se le pidió al paciente que se relajara, con los ojos cerrados, sin parpadeos, sin contacto dentario ni movimiento de los labios, y sin tragar. Se registró la actividad muscular en la posición de reposo. Luego se solicita a la paciente un apriete dentario máximo durante tres segundos, y se registraron los potenciales de acción de las unidades motoras reclutadas. Luego se solicita tres aprietes máximos de tres segundos cada uno con intervalos de dos segundos, y se registró la actividad sEMG. Finalmente se solicita deglución de saliva a la indicación del 
A

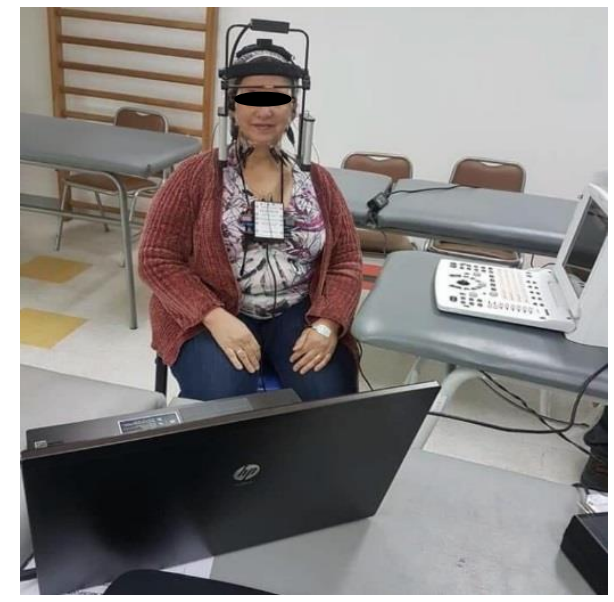

B

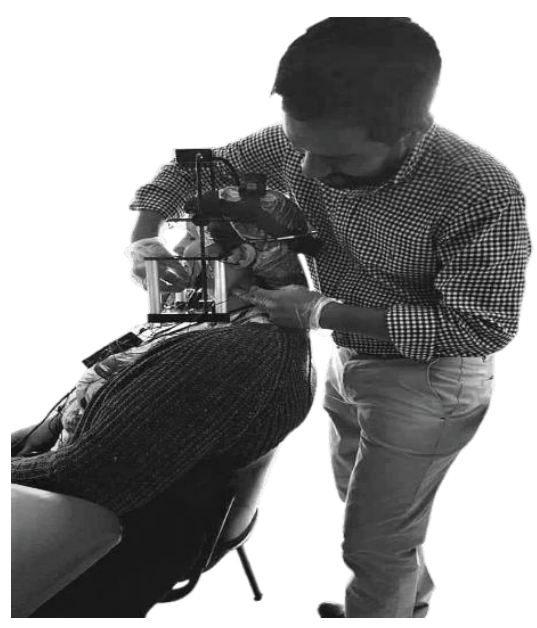

A. La configuración del dispositivo de adquisición, incluye amplificador conectado a software.

B. Técnica de punción Seca durante la electromiografía, que permite realizar ediciones fiables.

clínico y se registran los resultados sEMG correspondientes. La evaluación de los parámetros se llevó a cabo con la opción Summary Reviews (Software BIOPAK ${ }^{\mathrm{TM}}$ ), identificando sinergias y simetrías en los músculos revaluados (Tabla I).

Intervención (Figura 1): En una sola sesión, la PS con NMES se aplicó en los puntos gatillo latentes, con una corriente TENS con Pointer Plus $^{\mathrm{TM}}$, en una frecuencia de $10 \mathrm{~Hz}$, un ancho de pulso de 260 microsegundos y 2 miliamperios de intensidad, utilizando trenes de seis segundos tres veces en cada punto. Por decisión del clínico fueron puncionados los músculos pterigoideo lateral, pterigoideo medial $\mathrm{y}$ temporal anterior.

Evaluación post-punción: Los resultados biomecánicos se evaluaron inmediatamente después de aplicada la técnica de PS más NMES y seis días después del procedimiento. Respetando los mismos protocolos indicados por los fabricantes del equipo BIOPAK ${ }^{\mathrm{TM}}$, el mismo clínico que realizó la primera medición realiza los registros de esta etapa. Este investigador tiene más de 15 años de experiencia en el campo y más de diez años de experiencia en el uso de BIOPAK $^{\mathrm{TM}}$.

Hallazgos clínicos: Seis días después, la paciente reportó disminución subjetiva del dolor muscular y de la hiperactividad muscular. Hubo una mejora en los movimientos de la mandíbula, con un alivio de los síntomas de la columna cervical.

Cuando se compara la calidad del movimiento en diferentes momentos (T0=Línea de base; T1= inmediatamente después de $\mathrm{PS} ; \mathrm{T} 2=$ a 6 días después de PS), se observó una mejora en ROM (Figura 2) y velocidad del movimiento (Figura 3) justo después de PS más NMES comparando T1/T0, con la excepción de la velocidad máxima de apertura, apertura y cierre. Cuando se compara T2/T1, se notó una mejora en los parámetros alterados en T1/T0; la velocidad de cierre apareció disminuida. Finalmente, cuando se compara T2/T0, se encontró una mejora global en todos los parámetros (Tabla II). Además, se observó que los valores de inclinación de la apertura máxima disminuyeron después de PS más NMES, pero mejoraron una semana más tarde, y los valores de deflexión mejoraron inmediatamente después de PS y desaparecieron seis días después de la aplicación. En los valores absolutos, el tiempo de cierre mejoró inmediatamente después del PS y seis días después de esta, y los valores de cierre disminuyeron seis días después del PS más 


\section{NMES (Figura 2).}

JT-3D ${ }^{\mathrm{TM}}$ proporciona valores de referencia para determinar los movimientos de velocidad normales. Por lo tanto, cuando se compara la diferencia porcentual entre los hallazgos presentes y los del fabricante, se observó que el movimiento de apertura disminuyó antes de la PS más NMES (Figura 3). Es importante determinar que el lado izquierdo mejoró inmediatamente después de la intervención, y no hubo ninguna modificación en el lado derecho. Para el cierre, el efecto fue muy similar, con la normalización de la velocidad en el lado izquierdo, y una mejora en el lado derecho. Esto muestra una normalización de la velocidad de apertura y cierre en ambos lados, produciendo movimientos sincronizados. Esto se reveló cuando se observaron los parámetros de velocidad restantes, que mejoraron inmediatamente después de la PS más NMES y se estabilizaron seis días más tarde (Tabla III). Al analizar los valores absolutos, es evidente que inmediatamente después de PS más NMES, los movimientos mejoraron en los puntos de retorno vertical y anteroposterior, $\mathrm{y}$ mínimamente en los movimientos de retorno lateral, en ambos lados (Figura 3).

Comparación de los diferentes movimientos antes y 6-PPS (Tabla I)

Reposo: Hiperactividad del TA derecha, y simetría de los músculos de cierre mandibular. Hubo un reclutamiento de potenciales de acción de unidades motoras alterado en la sinergia entre los músculos TA y MM derechos. Seis días después, el TA derecho tenía una hiperactividad reducida, con simetría regular, y sinergia restaurada entre los músculos MM y TA derechos. Los otros músculos evaluados habían conservado sus valores.

Apriete x1: Cuando se pidió al paciente que hiciera apriete dentario máximo durante tres segundos, los músculos MMs se observaron agonistas de la acción con simetría regular. Hay muy buena simetría entre los TAs y muy baja sinergia entre el MM y TA del lado izquierdo. El lado derecho tenía muy buena sinergia. Buena simetría entre el ECOM y el músculo DA bilateralmente.
Tabla I: Valores electromiográficos en milivoltios de diferentes músculos, la diferencia absoluta entre T0 y T2 (ABS), y el porcentaje de cambio en los diferentes movimientos.

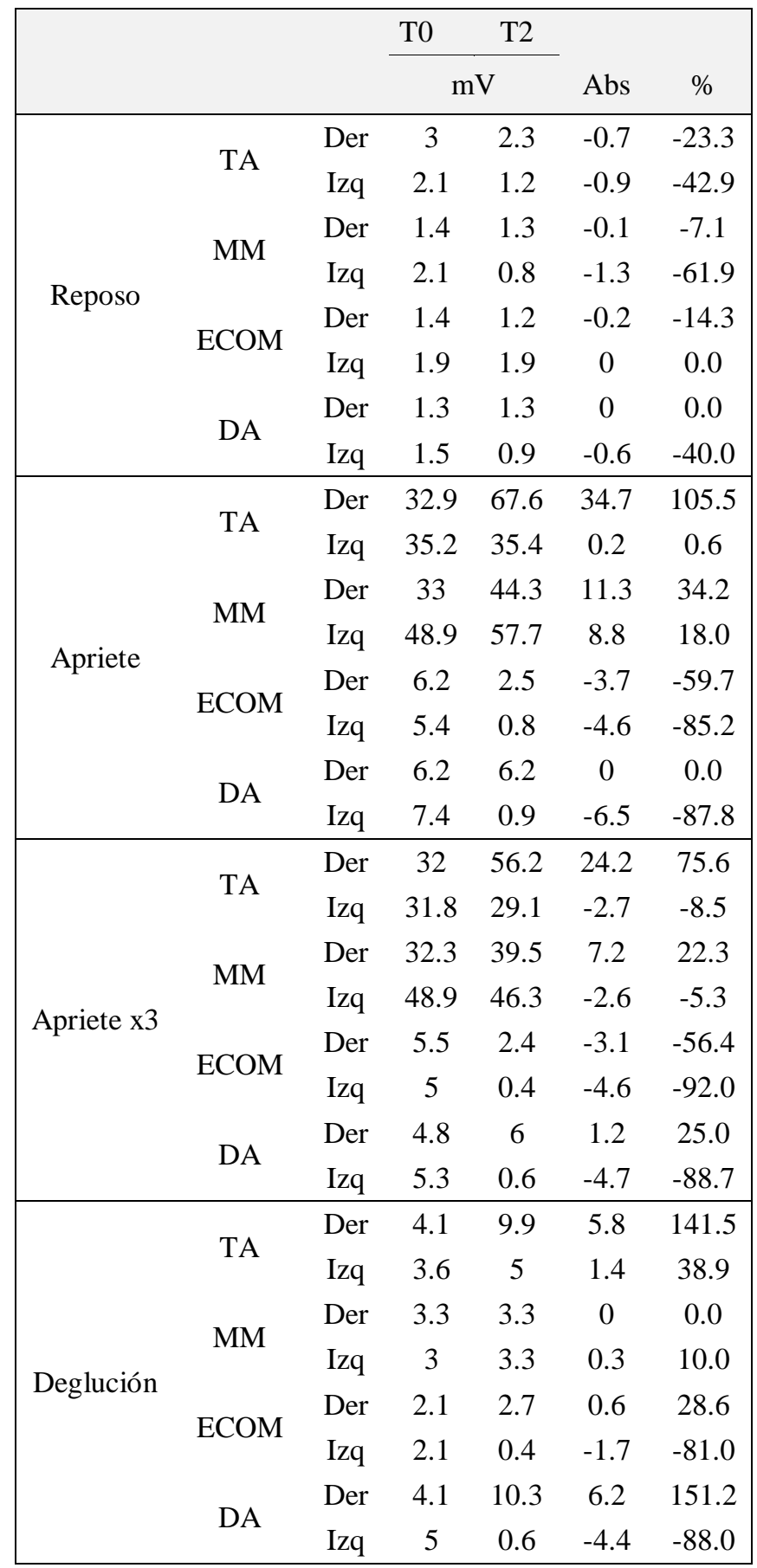

TA=Músculo Temporal Anterior; $\mathrm{MM}=$ Músculo de Masétero; ECOM= músculo esternocleidomastoideo; DA= Músculo Digástrico anterior; $\mathrm{mV}=$ Millivoltios; $\mathrm{ABS}=$ valor absoluto; T0= Línea de base/tiempo cero; T1= inmediatamente después de la punción seca/Tiempo uno; T2= 6 días después de la punción seca/Tiempo dos. Der $=$ derecho Izq= Izquierda 
Figura 2: Cambios en el rango de movimiento y la velocidad antes, justo después y 6 días después de la punción seca con NMES.

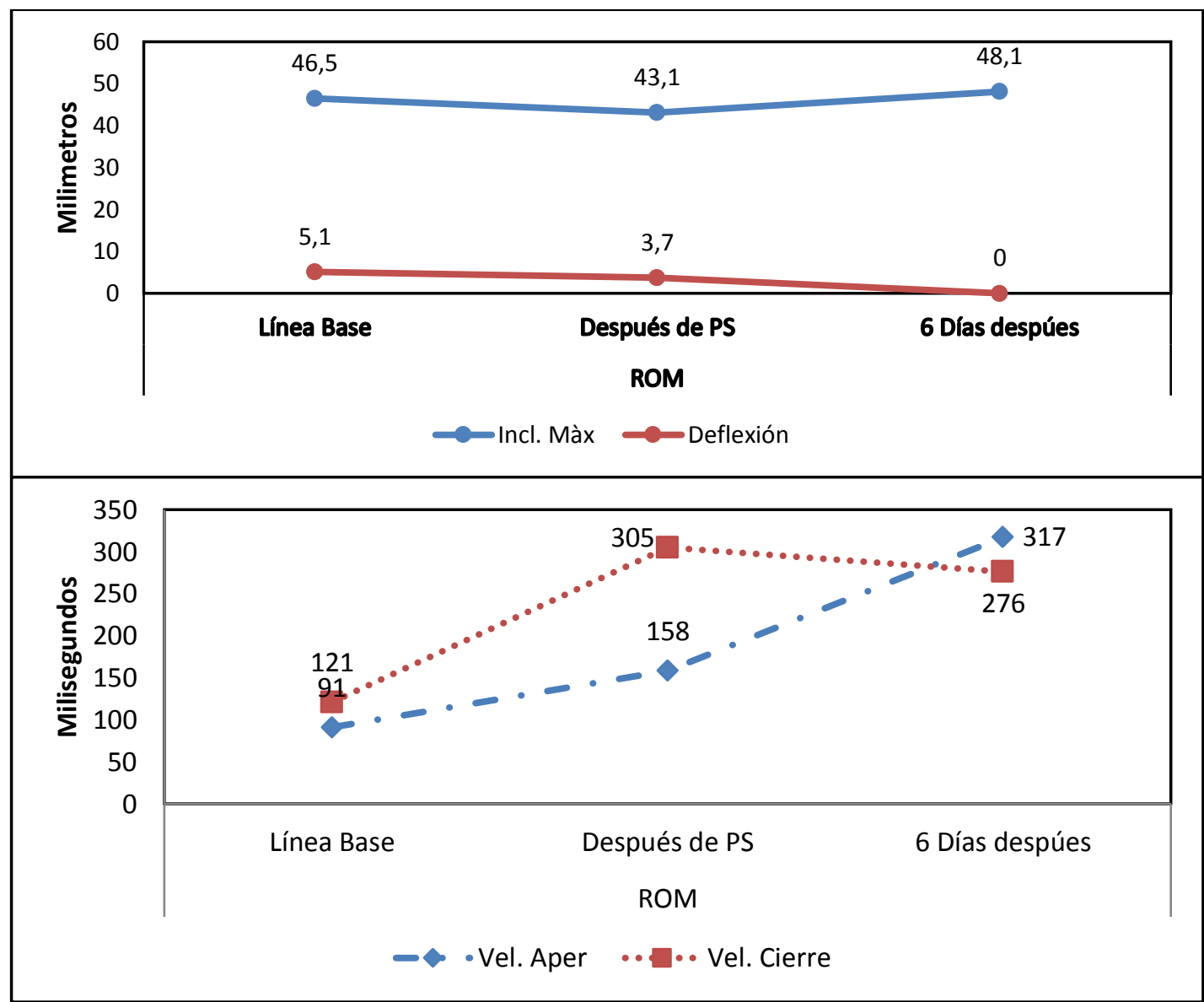

(A) Extensión de los movimientos de la deflexión y apertura. (B) Velocidades de apertura y cierre mandibular. ROM=Rango de movimiento; Incl= Inclinación; Vel=Velocidad

Tabla II: Valores porcentuales de las diferencias de respuesta de movimiento en reposo y velocidad. Antes de PS (T0), justo después (T1) y seis días después (T2).

\begin{tabular}{|c|c|c|c|c|}
\hline & & T1/T0\% & T2/T0\% & $\mathrm{T} 2 / \mathrm{T} 1 \%$ \\
\hline \multirow{4}{*}{ ROM } & Apertura Máxima & -7.3 & 3.4 & 11.6 \\
\hline & Vel. Apertura. & 73.6 & 248.4 & 100.6 \\
\hline & Vel Cierre. & 152.1 & 128.1 & -9.5 \\
\hline & Deflexión & -27.5 & -100 & -100 \\
\hline \multirow{4}{*}{ Velocidad } & Apertura Máxima & -9.9 & 0 & 11 \\
\hline & Vel. Apertura. & -27.7 & 5.3 & 45.7 \\
\hline & Vel Cierre. & -9.3 & 11 & 22.3 \\
\hline & Deflexión & -34.5 & -87.3 & -80.6 \\
\hline Patrón de apertura & $\begin{array}{l}\text { Dis } \\
\text { Bradi }\end{array}$ & \multicolumn{3}{|c|}{ Bradi (30mm Aper.) } \\
\hline Patrón de cierre & $\begin{array}{l}\text { Dis } \\
\text { Bradi }\end{array}$ & \multicolumn{3}{|c|}{ Bradi (10mm de sobremordida) } \\
\hline
\end{tabular}

DIS - Diskinesia; BRADI - Bradikinesia; ROM - Rango de movimiento; Vel - Velocidad 
Tabla III: Valores porcentuales de modificación en la velocidad durante la masticación en el lado izquierdo (L) y el lado derecho $(R)$ basado en los valores de referencia de JT-3D en milisegundos. También las diferencias producidas entre T1-TO, T2-TO y T2-T1

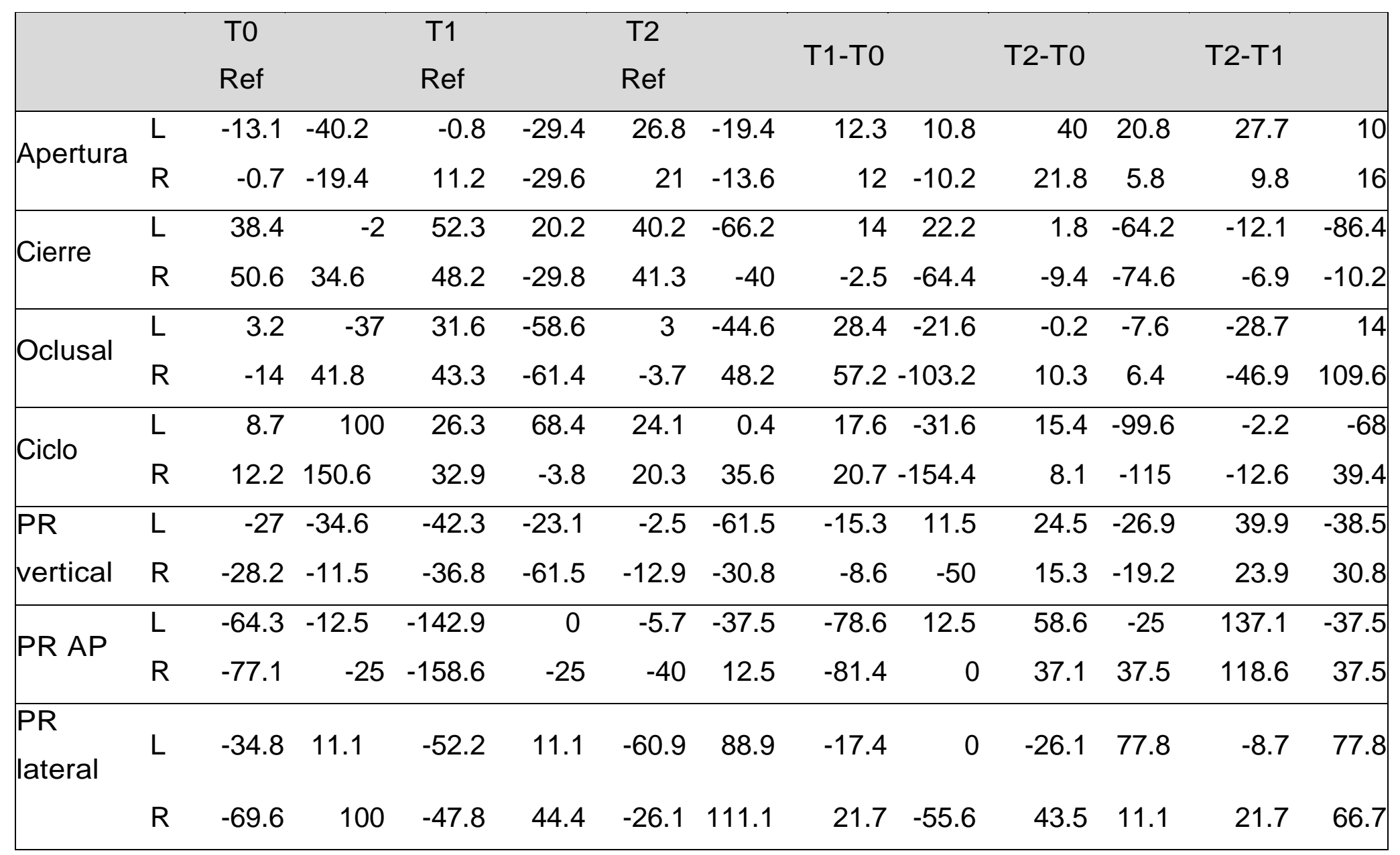

JT-3D - Análisis tridimensional, Trazador de Mandibular; PR - Punto de retorno; AP - Anterior/posterior; Ref - Valores de referencia; T0 - Línea de base/tiempo cero; T1 - inmediatamente después de la punción seca / Tiempo uno; T2 a 6 días después de la punción seca/Tiempo dos. 
Figura 3: Apertura y cierre con bolo de goma de mascar estandarizado antes, justo después y seis días después de la punción seca con NMES según lado de trabajo.

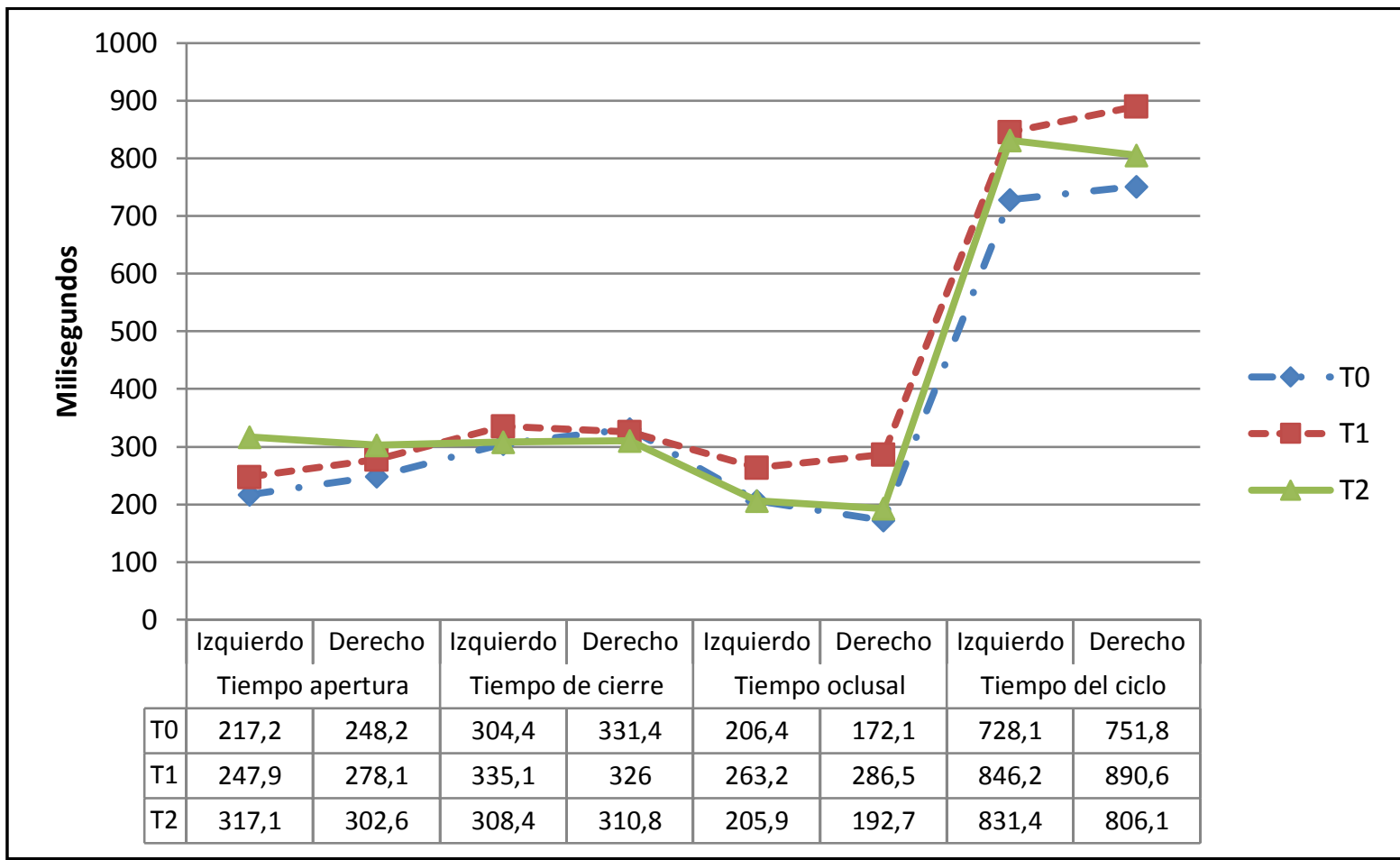

(A)

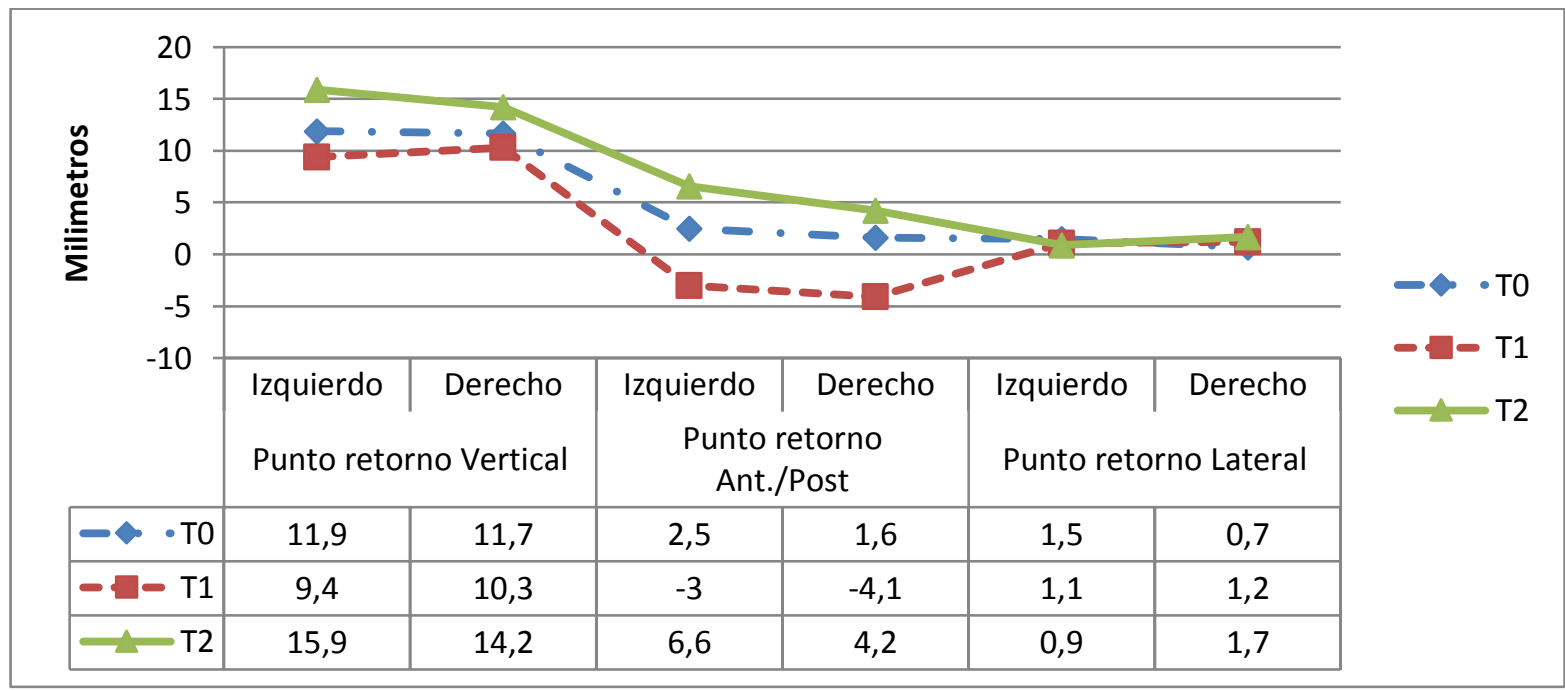

(B)

(a) Velocidades en milisegundos. (b) Distancias en milímetros. T0 =Línea de base/tiempo cero; T1=inmediatamente después de la punción seca / Tiempo uno; T2=seis días después de la punción seca/Tiempo dos.

En 6-PPS, la sinergia y la simetría entre los músculos MMs y TAs mejoraron a los valores normales. Los valores de reclutamiento fueron inferiores a lo habitual en esta acción. ECOM y DA en el lado derecho eran muy activos respecto al contralateral, lo que indica una simetría muy baja.

Apriete x3: Cuando se pidió al paciente que hiciera tres aprietes consecutivos (cada apriete de tres segundos con la fuerza máxima de mordida), se observa que los MMs se mantuvieron como agonistas con simetría regular. Había muy buena simetría entre TAs y una sinergia regular entre TA y MM en el lado izquierdo, pero era muy buena en el lado derecho. Había muy buena simetría en ECOMs y DAs. 6 días después, con 
mayor actividad electromiografía a la derecha, TA actuó como un agonista al apriete, produciendo simetría en el límite de ser regular. MM tenía muy buena simetría y sinergia en los límites de los valores normales. El ECOM derecho y el DA eran muy activos en comparación con el lado izquierdo, produciendo una simetría baja, pero con valores no significativos.

Deglución: Se pidió a la paciente que acumulara saliva en su boca, para que luego, a la orden del clínico, la tragara de una sola vez. En general, hubo un buen comportamiento muscular en las simetrías y sinergias. Seis días después, hubo una actividad muscular desequilibrada de DA en el lado derecho durante la deglución de saliva. MMs tenían muy buena simetría; Aunque TAs tenían menor simetría, era tan buena como MM. Hubo sinergia muy baja entre el TA y MM derechos. Esto indica que la lengua debe estudiarse en palatoposición, lo que explicaría el comportamiento de deglución con un movimiento aberrante de esta hacia el lado derecho.

ROM: Se pidió a la paciente que hiciera movimientos consecutivos de apertura y cierre a velocidad normal. Hubo una desviación de 5,1 mm a la izquierda en la apertura máxima y 2,2 $\mathrm{mm}$ a la derecha durante el cierre. Seis días después, se restauró la velocidad de apertura y cierre y se redujo la deflexión $2,2 \mathrm{~mm}$ a la izquierda y se aumentó $2,7 \mathrm{~mm}$ a la derecha.

Velocidad: Se solicitó a la paciente que realizara el mismo movimiento que ROM, pero a la velocidad máxima posible. Se registra la velocidad de apertura y cierre en los parámetros normales, con bradiquinesia y discinesia a los 30 mm desde la apertura hasta el final de la misma. Hubo una situación similar durante el cierre, con bradiquinesia a los $20 \mathrm{~mm}$ de esta, terminando con discinesia en los últimos $5 \mathrm{~mm}$. Seis días después de la PS más NMES, la bradiquinesia se controlaba durante la apertura y la discinesia se controlaba durante el cierre.

\section{DISCUSIÓN}

Seis días después de PS más NMES en los músculos pterigoideos laterales, mediales $\mathrm{y}$ maseteros superficiales, se encontró una mejora tanto en los parámetros biomecánicos como en la cadena sinérgica masticatoria. Estos resultados son similares a los de un estudio realizado en España $^{11}$, en el que trataron a 17 sujetos con TTM y aplicaron PS con puntos gatillos activos en MMs y TAs. Encontraron a una semana, mejoras inmediatas en el dolor, la sensibilidad, la apertura de mandibular y la discapacidad relacionada con la TTM, pero no lograron llegar al nivel de detalle biomecánico que se describe en el presente reporte. Otro estudio, también realizado en España ${ }^{12}$, se realizó en 48 pacientes con TTM crónico, donde la mitad de ellos recibieron PS sólo una vez a la semana durante tres semanas en el músculo pterigoideo lateral, mientras que la otra mitad recibió una combinación de methocarbamol/paracetamol cada seis horas durante tres semanas. Encontraron diferencias estadísticamente significativas en el dolor entre los grupos, y también una mejora en la apertura de la boca, la lateralidad y los movimientos de protrusiva desde el día uno en el grupo de tratamiento, como se encontró en el presente reporte. Estos resultados son interesantes porque PS es una técnica con un costo muy bajo y que produce los mismos efectos que inyecciones al $0.5 \%$ de lidocaína, como lo demuestra un estudio anterior en Brasil. ${ }^{13}$

Por otro lado, estos resultados son diferentes a los de otro informe de caso en Brasil ${ }^{14}$, donde utilizaron masaje, PS y terapia láser e informaron de una mejora de $10 \mathrm{~mm}$ para la apertura máxima. Esto se puede deber a que trataron pacientes con apertura restringida, que es diferente al paciente en este informe, que informó apertura normal, pero con dolor. Por lo tanto, cuando se comparan los resultados de EMG, está claro que reportan los mismos resultados que los del presente reporte, pero no pueden atribuirlo a ninguna de las alternativas terapéuticas. En este informe, la única intervención fue PS más NMES.

El equipo BIOPAK ${ }^{\mathrm{TM}}$ utilizado para registrar y medir los efectos de PS en este informe, también lo ha sido previamente por varios autores en la materia $^{15-17}$; Como se señaló anteriormente, el clínico a cargo de la medición tiene más de 10 años experiencia de uso del dispositivo, y más de 
15 años en el campo de la fisioterapia maxilofacial. Esto contribuyó a la producción de mediciones fiables. En el registro se utilizaron electrodos BioFLEX ${ }^{\mathrm{TM}}$ (BioResearch Associates, Inc., Milwaukee, WI), siguiendo el protocolo recomendado por el fabricante. Este protocolo incluye la limpieza de la piel del paciente con alcohol isopropílico para evitar cualquier efecto de maquillaje o loción. Para identificar los vientres musculares, se le pidió a la paciente que realizara apriete dentario máximo; el electrodo se localizó lejos de las unidades miotendinosas, paralelo a la orientación de la fibra muscular. Esto permite a los clínicos obtener mediciones confiables.

La actividad muscular óptima requiere la existencia de un buen equilibrio y coordinación entre cada uno de los grupos musculares (inhibición de antagonistas, coactivación de los sinergistas); ambas funciones están reguladas por el sistema nervioso central. Cualquier alteración funcional de las articulaciones causará tensiones musculares reflejas, que a su vez alterarán los patrones posturales y motores otra vez. El concepto de sinergia muscular o "sinergias musculares" son arreglos neuronales, donde el cerebro y la médula espinal simplifican el control motor de los músculos en unidades funcionales. Gracias a estas relaciones, el sistema neuromuscular se vuelve eficiente. La sinergia es fundamental en la estabilidad $y$ funcionalidad del aparato mandibular masticatorio y craneal. La pérdida de esto genera facilitación muscular con una respuesta espasmódica del músculo hiperactivo homólogo. Esta respuesta genera dolor y limitación funcional, incluida la presencia de puntos desencadenantes miofasciales. ${ }^{18}$ Esto último explicaría la decisión del clínico de puncionar el pterigoideo lateral sin haberlo evaluado, ya que dentro de la sinergia este músculo actúa a nivel del mismo centro motor. Y de la misma forma se explica la no valoración de este músculo en la electromiografía, ya que su modulación se ve reflejada en el resto de la musculatura mandibular.

Se encontró una mejora en la sinergia muscular seis días después de que se aplicó PS más NMES. El resto de los músculos mantuvieron valores normales, demostrando que esta mejora no se hizo a expensas de la compensación muscular o mecánica. Cuando se le pidió al paciente que realizara un apriete dentario máximo voluntario, los valores de reclutamiento de los potenciales de acción de unidades motoras se alteraron, o hubo un retraso en la coordinación de las órdenes musculares. Una razón para esto es el patrón motor alterado que estuvo presente durante mucho tiempo, lo que requiere más órdenes consecutivas para lograrlo. En el momento de realizar tres aprietes dentarios voluntarios máximos, separados por un período de descanso de dos segundos, los valores se normalizaron como resultado de este intento de adaptarse al nuevo engrama muscular en la masticación, sin dolor en la ATM. La velocidad, frecuencia $y$ trayectoria del movimiento mandibular ayudarían al clínico a determinar si el elemento terapéutico podría mejorar el rendimiento de la tarea.

Dentro de las limitaciones del estudio se puede encontrar la ausencia de objetivación del dolor, y la ausencia de diagnóstico específico del TTM, ahora bien, el objetivo de describir los cambios de la biomecánica y la cadena sinérgica muscular masticatoria a través de la medición biométrica con el equipo BIOPAK ${ }^{\mathrm{TM}}$, genera un aporte relevante y que debe ser considerado para futuras investigaciones.

\section{CONCLUSIÓN}

Una sola sesión de PS más NMES en los músculos pterigoides laterales y mediales y la parte anterior de los músculos temporales mostró una mejor respuesta electromiográfica y estabilidad biomecánica de la ATM en un paciente con una larga historia de signos y síntomas. Esta mejora se mantuvo durante seis días. El uso del BIOPAKTM resulta de utilidad clínica, proporcionando registros y evaluaciones objetivas del efecto terapéutico de sus intervenciones.

\section{CONFLICTOS DE INTERÉS}

Los autores declaran que no existe conflicto de intereses ni financiación para el presente informe de caso. 


\section{AGRADECIMIENTOS}

Los autores desean agradecer a Manuel Olmedo PT MSc por su contribución en la aplicación de PS más NMES al paciente en este informe y al Dr. Mariano Rocabado Seaton por facilitar el equipo BIOPAK ${ }^{\mathrm{TM}}$.

\section{REFERENCIAS}

[1] Mujakperuo HR, Watson M, Morrison R, Macfarlane TV. Pharmacological interventions for pain in patients with temporomandibular disorders. Cochrane Database Syst Rev. 2010 Oct;(10):CD004715.

[2] Schiffman EL, Truelove EL, Ohrbach R, Anderson GC, John MT, List T, et al. The Research Diagnostic Criteria for Temporomandibular Disorders. I: overview and methodology for assessment of validity. J Orofac Pain. 2010;24(1):7-24.

[3] Leskinen J, Suvinen T, Teerijoki-Oksa T, Kemppainen P, Napankangas R, Alstergren P, et al. Diagnostic criteria for temporomandibular disorders (DC/TMD): inter-examiner reliability of the Finnish version of Axis I clinical diagnoses. J Oral Rehabil. 2017 Jul;44(7):493-499.

[4] Bianchini EM, Paiva G, de Andrade CR. Mandibular movement patterns during speech in subjects with temporomandibular disorders and in asymptomatic individuals. Cranio. 2008 Jan;26(1):50-58.

[5] Cao Y, Zhang W, Yap AU, Xie QF, Fu KY. Clinical characteristics of lateral pterygoid myospasm: a retrospective study of 18 patients. Oral Surg Oral Med Oral Pathol Oral Radiol. 2012 Jun;113(6):762-765.

[6] Macedo SAMMSH CR, Prado G. Occlusal splints for treating sleep bruxism (tooth grinding). Cochrane Database of Systematic Reviews. 2007;(4). Available from: https://doi.org//10.1002/14651858.CD005514 .pub2.

[7] Luther F, Layton S, McDonald F. Orthodontics for treating temporomandibular joint (TMJ) disorders. Cochrane Database Syst Rev. 2010 Jul;(7):CD006541.

[8] Luther F, Layton S, McDonald F. WITHDRAWN: Orthodontics for treating temporomandibular joint (TMJ) disorders. Cochrane Database Syst Rev. 2016 Jan;(1):CD006541.

[9] Liu L, Huang QM, Liu QG, Thitham N, Li LH, Ma YT, et al. Evidence for dry needling in the management of myofascial trigger points associated with low back pain: A systematic review and meta-analysis. Arch Phys Med Rehabil. 2018 01;99(1):144-152.

[10] Vier C, Almeida MB, Neves ML, Santos ARSD, Bracht MA. The effectiveness of dry needling for patients with orofacial pain associated with temporomandibular dysfunction: a systematic review and metaanalysis. Braz J Phys Ther. 2018 Aug.

[11] Blasco-Bonora PM, Martin-Pintado-Zugasti A. Effects of myofascial trigger point dry needling in patients with sleep bruxism and temporomandibular disorders: a prospective case series. Acupunct Med. 2017 Mar;35(1):69-74.

[12] Gonzalez-Perez LM, Infante-Cossio P, Granados-Nunez M, Urresti-Lopez FJ, LopezMartos R, Ruiz-Canela-Mendez P. Deep dry needling of trigger points located in the lateral pterygoid muscle: efficacy and safety of treatment for management of myofascial pain and temporomandibular dysfunction. Medicina Oral, Patologia Oral and Cirugia Bucal. 2015;20(3):e326.

[13] Silva ROFd, Conti PCR, Arau jo CdRP, Silva RdS. Evaluation of dry needling and $0.5 \%$ lidocaine injection therapies in myofascial pain trigger points in masticatory muscles. Dental Press J Orthod. 2012;17(2):113-118.

[14] Pessoa DR, Costa DR, Prianti BM, Costa DR, Delpasso CA, Arisawa EALS, et al. Association of facial massage, dry needling, and laser therapy in Temporomandibular Disorder: case report. Codas. 2018 Nov;30(6):e20170265.

[15] $\mathrm{Ku} \mathrm{J}$, Szarejko KD, Aleksandrowicz K, Gobiewska M. The role of soft tissue mobilization in reducing orofacial and general complaints in a patient with Kimmerle anomaly and temporomandibular joint disorder: A case report. Cranio. 2019 Jan;1-14.

[16] Wieczorek A, Loster J, Loster BW. Relationship between occlusal force distribution and the activity of masseter and anterior temporalis muscles in asymptomatic young adults. Biomed Res Int. 2013;2013:354017.

[17] Sharma S, Crow HC, Kartha K, McCall WD, Gonzalez YM. Reliability and diagnostic validity of a joint vibration analysis device. BMC Oral Health. 2017 Feb;17(1):56.

[18] Rana M, Yani MS, Asavasopon S, Fisher BE, Kutch JJ. Brain Connectivity Associated with Muscle Synergies in Humans. J Neurosci. 2015 Nov;35(44):14708-14716. 


\section{CÓMO CITAR ESTE ARTÍCULO}

Espinoza S; Marfull N. Cambios en la dinámica mandibular y en la cadena sinérgica muscular mandibular posterior a la punción seca y TENS en paciente con TTM. Appli. Sci. Dent. 2020; 1(1); 6373. DOI: $10.22370 /$ asd.2020.1.1.2081 